\title{
ARTICLES
}

\section{L'engagement et la responsabilité des interprètes en milieu social : les limitations de leur pouvoir} Agency and Responsibility of Community Interpreters:
Their Empowerment Limitations

\author{
Iwona Kasperska \\ Adam Mickiewicz University de Poznań \\ iwona.kasperska@amu.edu.pl
}

\begin{abstract}
This paper discusses the agency of community interpreters in a cross-cultural environment. Their empowerment is determined by several linguistic and extralinguistic factors, such as: mastering the language of communication by the speakers, social status of the languages used in multilingual countries, other parties' behaviour, interlocutors' different cultural backgrounds, interpreter's loyalties (to the client and/or to the institution), and social norms of the speakers. This study deals with a number of communicative, social and pragmatic features, as well as it reveals the level of the interpreters' power and responsibility for a successful outcome of communication. The main conclusion is that, even if traditionally they are depicted as a mere language conduits, neutral and invisible, the interpreters in social services turn out to be very visible actors. Their agency is flexible, e.i. it changes depending on the particular interaction, and their empowerment consists in making every communication as easy as possible for people deprived of such the agency.
\end{abstract}

Keywords: interpreting for social services, interpreter's agency, interpreter's empowerment, linguistic and extralinguistic factors, interpreter's visibility

\section{INTRODUCTION}

L'acte d'interprétation en milieu social implique la participation de trois parties : le client, le récepteur et l'interprète. La relation qui s'établit entre ces acteurs reflète l'asymétrie de leur pouvoir et le hiatus culturel (Valero-Garcés \& Martin, 2008), 
l'asymétrie des connaissances dont ils disposent (Tryuk, 2004 ; Klimkiewicz, 2005) et l'asymétrie en connaissance du milieu professionnel. Dans un contexte institutionnel où ce type de communication a normalement lieu, la relation en question est souvent régie par des règlements internes ou des lois officielles. En outre, les rôles et le statut de l'interprète peuvent être dynamiques et changer en fonction des comportements imprévus des participants à l'échange, qui n'est pas seulement d'ordre linguistique. Comme le signalent Valero-Garcés et Martin (2008), l'interprétation en milieu social signifie aussi l'urgence de communiquer, le manque de sources « textuelles ", l'absence de profil professionnel de l'interprète communautaire, l'interaction dans une situation dramatique pour l'une des parties, le caractère imprévisible du déroulement de ce type de communication et le possible changement des objectifs communicationnels d'une rencontre à l'autre. Tous ces traits caractéristiques induisent chez les interprètes la prise de décisions variées dévoilant le degré de leur engagement et de leur responsabilité, ou bien les limitations de leur pouvoir.

Dans les lignes qui suivent, nous voudrions aborder le problème du pouvoir de l'interprète en tant qu'acteur social, en nous basant sur notre expérience comme théoricienne et praticienne de l'interprétation et en recourant aux contributions et expériences des autres auteurs, notamment Wadensjö (1998), Angelelli (2004), Barsky (2005), Tryuk (2004, 2006), Kleinert (2016), Martin et Abril Martí (2008) et Määttä (2017), entre autres. Notre objectif est de décrire et d'explorer quelques facteurs linguistiques et extralinguistiques qui, selon nous, pèsent sur le travail de l'interprète, car il exige une médiation entre différents pouvoirs, intérêts et compétences. Dans la révision des exemples d'interactions réelles nous chercherons à éclairer le degré d'engagement de l'interprète et de sa " volonté et capacité d'agir » (Buzelin, 2011) aux différents niveaux de la médiation communicationnelle que l'interprète est censée faciliter.

\section{LE POUVOIR DE L'INTERPRÈTE : QUELQUES REMARQUES PRÉLIMINAIRES}

Dans son étude Empowerment and Community Planning, Elisheva Sadan (1997) rappelle que l'intérêt autour de l'«acquisition du pouvoir» («empowerment») comme un phénomène inscrit dans un large contexte social provient de la nécessité de doter de pouvoir les sujets sociaux qui n'en disposent pas et qui ne sont donc pas capables de résoudre leurs problèmes liés, entre autres, aux services sociaux. Sadan le définit comme «une transition de l'état d'impuissance vers celui de plus grand contrôle sur sa propre vie, son sort et son environnement $»^{1}$ (1997, p. 13). La chercheuse israélienne met en relief trois contextes ou, comme elle le veut, trois dimen-

\footnotetext{
${ }^{1}$ « $[\ldots]$ a transition from a state of powerlessness to a state of more control over one's life, fate, and environment » (Sadan, 1997, p. 13). Toutes les traductions des citations sont les nôtres.
} 
sions de la condition sociale auxquelles le processus de l'acquisition du pouvoir s'adresse : les sentiments et les capacités des gens, la vie en collectivité à laquelle ils appartiennent, la pratique professionnelle qui leur permettra d'atteindre un pouvoir. Il s'avère que le contexte de l'interprétation en milieu social met en évidence les trois dimensions mentionnées par Sadan. Premièrement, il s'agit de la situation personnelle d'un sujet qui, par exemple, est soumis à la comparution devant un tribunal ou recourt à une consultation médicale; deuxièmement, chaque individu (soit l'interprète, soit les intervenants) appartient à une collectivité ethnique ou sociale dont la vie est régie par les lois; et troisièmement, l'interprétation est une pratique professionnelle qui se déroule inévitablement dans un contexte institutionnel et, donc, collectif par excellence.

Pour Maria Tymoczko (2007, p. 216), le pouvoir du traducteur est lié directement à l'idéologie. Du point de vue de la théorie postcoloniale, ce pouvoir commence au moment où il faut prendre une décision concernant chaque étape du processus de traduction : à commencer par le choix du texte à traduire pour passer à la sélection des techniques de traduction des unités les plus simples. Bien que le traducteur ne soit pas activiste, la nature de son travail implique un pouvoir à cause de l'éthique et de l'idéologie qui sont inscrites dans les choix qu'il fait. C'est par l'action que le traducteur peut intervenir dans le processus de traduction. L'action s'avère un motclé, parce que l'activisme appelle plutôt à un engagement proactif qu'à une résistance qui est réactive. Tymoczko explique que, dans le contexte postcolonial, les traducteurs engagés « sont visibles comme sujets et en tant qu'agents ils ont des objectifs politiques à réaliser par l'intermédiaire des traductions » (2007, p. 213).

Le deuxième point que nous voudrions souligner c'est que l'idée de la transparence ou l'invisibilité de l'interprète en milieu social n'a rien à voir avec la réalité professionnelle. C'est une conclusion à laquelle sont déjà arrivés de nombreux chercheurs (Angelelli, 2004 ; Barsky, 2005 ; Tryuk, 2006 ; Valero-Garcés \& Martin, 2008 ; Springer, 2010 ; Kruk-Junger, 2013) qui ont réalisé des projets dans les institutions offrant des services ou qui ont pu interviewer des interprètes communautaires. Franz Pöchhacker (2004, p. 147) souligne un paradoxe dans l'image de l'interprète vu jadis comme intermédiaire, guide ou négociateur et dont l'activité, au $\mathrm{XX}^{\mathrm{e}}$ siècle, a été codifiée et circonscrite dans le cadre de l'éthique professionnelle. Pour nous, l'interprète par le fait même d'être physiquement présent n'est, pour le moins, pas transparent, c'est un actant muni du pouvoir de médiateur lui permettant d'influencer le déroulement de la communication. Ce pouvoir est, bien évidemment, soumis à des contraintes à caractère linguistique, culturel et social, que nous évoquerons ultérieurement. Nous signalons pourtant qu'il est impossible de traiter l'interprète comme un conduit neutre tandis qu'il est un être humain confronté à une interaction avec un autre sujet humain.

En considérant l'interprète comme celui dont le travail a un impact réel sur le succès en communication nous partageons le point de vue de plusieurs chercheuses 
et chercheurs, entre autres Angelelli (2003, 2004), Klimkiewicz (2005), Barsky (2005) et Valero-Garcés et Martin (2008). Robert F. Barsky prône explicitement la « traduction activiste» («activist translation»), en se référant également et sans aucun doute à l'interprétation communautaire lorsqu'il affirme que « les traducteurs devraient être impliqués et engagés, au-delà de l'acte de remplacer une unité lexicale par une autre $»^{2}$ (2005, pp. 17-18). En citant une panoplie d'exemples d'abus de pouvoir commis envers des immigrés illégaux, observés au sein des institutions américaines, l'auteur rappelle que les traducteurs/interprètes ne sont pas censés être activistes. Bien au contraire, ils sont «explicitement encouragés à être exclusivement impartiaux $»^{3}$ (Barsky, 2005, p. 18). Cette attitude de Barsky renvoie immédiatement à notre troisième point, c'est-à-dire aux limites de l'éthique professionnelle. Son cadre est assez flou parce qu'il est très difficile de définir le degré auquel l'interprète peut intervenir dans l'interaction entre le client et le récepteur et les codes de déontologie et d'éthique ne le précisent pas. Nous y réfléchirons cidessous, en prenant comme référence la perspective de l'interprète en tant que participant actif à la communication, c'est-à-dire celui qui ne considère pas son rôle comme un conduit neutre.

Pour conclure cette partie, nous aimerions rappeler comment Michel Foucault entend le concept du pouvoir :

Par le pouvoir, il me semble qu'il faut comprendre d'abord la multiplicité des rapports de force qui sont immanents au domaine où ils s'exercent, et sont constitutifs de leur organisation; le jeu qui par voie de luttes et d'affrontements incessants les transforme, les renforce, les inverse ; les appuis que ces rapports de force trouvent les uns dans les autres, de manière à former chaîne ou système, ou, au contraire, les décalages, les contradictions qui les isolent les uns des autres; les stratégies enfin desquelles ils prennent effets, et dont le dessin général ou la cristallisation institutionnelle prennent corps dans les appareils étatiques, dans la formulation de la loi ; dans les hégémonies sociales (1976, pp. 121-122).

Dans cette description foucaultienne du pouvoir se reflètent les « rapports de force » typiques de l'interprétation communautaire étant donné son caractère d'affrontement entre des individus et des institutions. C'est la situation dans laquelle l'interprète se trouve confronté aux intérêts des uns et des autres et contraint de résoudre un problème de loyauté professionnelle ou éthique. À propos de la « situation », Foucault prône que le pouvoir est justement le nom donné à « une situation stratégique complexe dans une société donnée » (1976, p. 123). Sans aucun doute, l'interaction en interprétation communautaire, se produisant entre les actants représentant des individus ou des institutions, répond aux paramètres de ladite situation.

\footnotetext{
${ }^{2}$ « [...] translators ought to be involved, engaged, over and above this act of substituting one lexical item for another » (Barsky, 2005, pp. 17-18).

${ }^{3}$ « [...] explicitly discouraged from being anything other than impartial» (Barsky, 2005, p. 18).
} 
Dans les deux sous-chapitres qui suivent nous nous occuperons des facteurs linguistiques et extraliguistiques qui jouent un rôle très important en interprétation communautaire. Sandra Beatriz Hale (2007), par exemple, propose un classement des défis auxquels les interprètes doivent faire face, en les regroupant en quatre catégories, à savoir : l'interprétation telle quelle (langues, compétences de l'interprète, contraintes temporelles, culture), le contexte (conditions de travail, information insuffisante pour l'interprète, contraintes liées à la situation), les participants (attitudes négatives des usagers des services d'interprétation, incompréhension du processus d'interprétation par les participants, incompréhension du rôle de l'interprète, méconnaissance des besoins professionnels de l'interprète) et enfin, le système (formations insuffisantes, manque d'exigences quant au recrutement des interprètes, manque de support de la part des institutions, rémunération insuffisante). En tenant compte du fait que, parfois, il est impossible de séparer le linguistique du culturel, du social et du politique (ce qui d'ailleurs n'est pas notre but ici), nous ferons référence à plusieurs problèmes mentionnés ci-dessus.

\section{LES FACTEURS LINGUISTIQUES}

Parmi les facteurs linguistiques qui nous intéressent figurent le niveau de connaissance de la langue de communication par les participants à l'interaction et le statut des langues utilisées par les intervenants. La première situation se produit lorsque la langue de communication est une L2 pour au moins un des intervenants, c'est-à-dire : une langue étrangère apprise dans le contexte scolaire, ou utilisée comme langue de communication dans les pays plurilingues. Exemple : l'espagnol au Mexique ou l'anglais aux États-Unis. L'intervenant contraint alors d'utiliser la langue de communication, qui n'est pas sa langue native, risque de se retrouver en position défavorable. Profitant de son expérience d'interprète communautaire et de celles de ses collègues en Finlande, Simo K. Määttä (2017) constate qu'au moins $80 \%$ des interprétations dans le contexte d'immigration dans le Grand Helsinki s'effectuent en anglais comme lingua franca. La plupart des interprètes sont aussi des usagers de l'anglais comme L2.

Dans la situation concrète analysée par Määttä (2017), l'interprète et l'agent de l'État étaient des natifs parlant le finnois alors que la personne interrogée était originaire d'un pays dans lequel l'anglais était la langue officielle et la plus importante langue de communication. L'analyse a révélé que l'emploi de l'anglais avait exigé de l'interprète encore plus de médiation linguistique et interculturelle, allant au-delà d'une « simple» interprétation. Les normes stratégiques «normalement» appliquées se sont avérées difficiles à maintenir, particulièrement à cause de la modalité de l'interprétation, à savoir : l'interprétation téléphonique. Le fait de se servir d'une troisième langue a impliqué des contraintes linguistiques, comme l'accent et 
la prononciation spécifiques des participants; les contraintes culturelles, par exemple : les différents systèmes scolaires dont la structure a été présentée par le biais d'une troisième culture ; et les procédés d'interprétation, comme le besoin de se servir plus souvent de reformulations.

$\mathrm{Au}$ travers des techniques de reformulation et de vérification, en plus des corrections constantes de l'interprète, décrites par Määttä (2017), nous pouvons observer l'effort fourni pour garantir le succès communicationnel. L'engagement et la responsabilité de l'interprète sont également notables au niveau des droits linguistiques de la personne interrogée car, comme le chercheur finnois le souligne, l'interprète vérifiait systématiquement sa compréhension des répliques et, après l'interview, se souciait de la correction de la transcription de l'entretien. Les mêmes observations ont été notées par Rafał Springer (2010) dans sa recherche effectuée à l'Office des étrangers en Pologne : certains interprètes vérifiaient systématiquement la transcription de l'entretien faite par le fonctionnaire afin de le compléter, corriger et confirmer l'orthographe des noms propres. La pratique montre qu'au cours de l'interprétation, la construction du sens est dynamique. Celle-ci s'effectue par des questions additionnelles posées par/aux participants à la conversation, par des éclaircissements souhaités et apportés, des répétitions, des paraphrases, des explications ajoutées et par des récapitulations, c'est-à-dire une série de procédés grâce auxquels l'interprète est visible (Angelelli, 2004). Dans l'attitude de l'interprète décrite par Määttä (2017), le degré de l'acquisition du pouvoir était manifestement très élevé.

Pour Määttä, recourir à l'anglais comme lingua franca dans un entretien rend le « multilinguisme invisible et ainsi dissimule l'asymétrie des pouvoirs, implicite dans chaque contexte multilingue complexe ${ }^{4}(2017$, p. 41). Cet effacement d'une langue (non révélée par Määttä dans son article) dans la communication interculturelle par excellence, dévoile l'autre problème linguistique qui nous intéresse, c'est-à-dire : le statut des langues utilisées. Dans le contexte de l'interprétation communautaire, c'est le cas des langues de moindre diffusion pour lesquelles il n'y a pas d'interprètes qualifiés, ou de celle des langues des immigrés/résidents pour l'interprétation desquelles il est « suffisant » d'employer un interprète naturel, natif (Harris, 1976), ad hoc ou pratique (Kleinert, 2016) à défaut d'interprète professionnelle. Réfléchissons sur les enjeux d'un tel choix.

L'interprète naturel est un individu bilingue qui sert de médiateur linguistique et interculturel dans différentes situations formelles et informelles de la vie quotidienne, activité pour laquelle il ne reçoit très souvent aucune rémunération (Antonini, 2011). Dans le contexte mexicain que nous commenterons, le manque de rémunération et le bilinguisme asymétrique ne constitue qu'une facette du problème. La notion d'interprète naturel, appliquée souvent en référence aux enfants qui

\footnotetext{
${ }^{4}$ « $[\ldots]$ multilingualism invisible and therefore also disguises the power imbalance inherent in any complex multilingual context » (Määttä, 2017, p. 41).
} 
accompagnent les parents monolingues dans les services sociaux, a de fortes connotations coloniales (Kleinert, 2016) au Mexique, où l'on adopte toujours une attitude fortement paternaliste envers la population indigène. L'adjectif naturel renvoie immédiatement à la catégorie coloniale des habitants naturels du continent américain, auxquels on refusait l'intelligence, les connaissances, l'autonomie et l'autodétermination. Bien que certains de ces traits caractéristiques (comme le droit à l'auto-détermination) n'étaient pas encore connus à l'époque coloniale, dans le Mexique d'aujourd'hui, la disposition envers les indigènes traités comme «bons sauvages » reste toujours bien présente.

Le recours aux interprètes ad hoc est considérée non seulement dangereux pour les interprètes comme catégorie professionnelle, mais aussi pour les parties intéressées qui, consécutivement à une interprétation incorrecte, peuvent souffrir de suites fâcheuses. Cette situation n'est pas l'apanage des pays dans lesquels les services d'interprétation sont peu développés ou limités suite à la situation économique ou culturelle, comme au Mexique par exemple (Kasperska, 2016a, 2016b ; Kleinert, 2016). Le recours au service d'interprète ad hoc s'avère aussi une caractéristique culturelle parmi les immigrés qui font plus confiance aux membres de leur famille (bien qu'ils soient parfois très jeunes), ou aux membres de la même communauté ethnique maîtrisant la langue du pays d'accueil, qu'aux autochtones (Atonini, 2011). Małgorzata Tryuk (2006) et Cristina V. Kleinert (2016) citent l'exemple d'institutions respectivement polonaises et mexicaines au sein desquelles des employés connaissant une langue étrangère qui, parfois, n'est pas la langue en question mais une lingua franca (Pologne) ou comprenant à peine une langue indigène qui n'est pas la même variante dialectale que la leur (Mexique) servent d'interprètes ad hoc lorsqu'il s'agit de faciliter la communication « au niveau de base ». Selon nous, cette attitude de la part des institutions n'est pas neutre pour trois raisons principales : elle illustre le faible statut dont «jouit» la L1 de la personne vulnérable en Pologne et au Mexique ; elle montre à quel point les droits linguistiques de la personne concernée sont négligés, voire violés ; et finalement, met en évidence le peu d'importance que les pouvoirs publics attachent aux compétences d'interprétation. La supposée suprématie des institutions représentant « le système » supprime même l'espace dans lequel l'interprète professionnelle devrait exercer sa profession. Ainsi, il est douteux qu'un interprète ad hoc ait une influence sur le déroulement de l'interaction en tant que telle, parce le pouvoir dont il dispose est, en réalité, celui de l'employé de l'État.

L'interprétation en milieu social au Mexique est un exemple très particulier de la médiation interlinguistique et interculturelle car on a affaire non seulement aux émigrés ou aux résidents étrangers recourant à ce service mais aussi aux Mexicains près de 7,4 millions de la population, selon l'INEGI (2015) - dont la première langue n'est pas l'espagnol mais l'une des soixante-huit langues vernaculaires bénéficiant du statut officiel de langues nationales. Ce sont eux qui, dans leur propre pays, peuvent avoir besoin de l'interprète et, plus précisément, ont le droit de le 
revendiquer. C'est la Constitution politique des États-Unis mexicains qui le garantit. Ce droit n'est hélas très souvent garanti que «sur le papier ». Comme le précise Kleinert (2016), il y a quelques années, le Mexique a initié une réforme du système juridique censée donner plus d'espace aux droits linguistiques des indigènes et, à la fois, aux interprètes des langues nationales, autres que l'espagnol. Il faudrait que l'État reconnaisse de façon permanente son obligation et non pas seulement selon son bon vouloir. Le cas de Jacinta Francisco Marcial, femme d'origine otomí (ou hñähñú), accusée d'avoir, avec deux autres femmes, séquestré six agents de la police fédérale, a été condamnée à 21 ans de prison sans que son droit à l'interrogation en otomí ait été respecté. Le problème est que, selon la Commission nationale pour le développement des ethnies indigènes (en esp. Comisión Nacional para el Desarrollo de los Pueblos Indígenas), le niveau de connaissance de l'espagnol de Jacinta Francisco Marcial, l'année de sa détention en 2005, n'atteignait que 20\%. Après trois ans de prison, son dossier a été réexaminé et, d'après une autre institution : l'Institut national des langues indigènes (en esp. Instituto Nacional de Lenguas Indígenas), ses droits linguistiques, y compris son droit à l'interprète, ont été violés (Kleinert, 2016 ; Centro Prodh, 2018). Cet exemple emblématique, qui n'est toutefois pas unique en son genre, illustre jusqu'où peut aboutir la négligence du droit à l'interprète, bien évidemment professionnelle. Il démontre également le pouvoir total de l'État sur le sort de sa citoyenne, une femme vulnérable, non protégée, dépourvue de ses droits en tant que personne. Selon notre expérience personnelle et nos recherches (Kasperska, 2015, 2016b) dédiées à la formation des traducteurs et des interprètes menées au Mexique, les interprètes des langues vernaculaires accrédités sont des indigènes et des métis, c'est-à-dire les personnes appartenant à des groupes aussi vulnérables que celui de Jacinta Francisco Marcial. La situation linguistique au Mexique requiert le même activisme dont parle Barsky (2007) et un réel engagement de l'interprète, ce qui, par ailleurs, a été souligné pendant la «Première rencontre nationale des interprètes des langues indigènes », célébrée les 24 et 25 octobre 2013, à Oaxaca, Mexique.

\section{LES FACTEURS EXTRALINGUISTIQUES}

Les facteurs extralinguistiques qui affectent l'interprétation peuvent être d'ordre socio-communicatif, contextuel, fonctionnel et pragmatique. Par exemple, les tensions interethniques sur lesquelles doit "agir » l'interprète pour les éteindre, les réactions à la situation de stress, la perception du pouvoir et le pouvoir réel des participants à l'interaction, la loyauté envers le commanditaire ou le client, ou encore, le comportement des intervenants sont quelques exemples de la complexité situationnelle dont parle Foucault (1976). Il faut mentionner aussi les valeurs morales, les normes personnelles et culturelles, c'est-à-dire, les différences indivi- 
duelles entre les participants à l'interaction, ainsi que la situation de communication, qui ne peuvent pas être ignorées si on veut qu'elle réussisse. Angelelli le décrit de la manière suivante :

Si la communication est censée réussir, l'interprète doit aussi faire attention aux facteurs sociaux qui affectent la communication, à savoir : les origines sociales des interlocuteurs ou les contraintes imposées par la situation dans laquelle l'évènement communicatif interprété (ECI) a lieu $(2004, \text { p. } 7)^{5}$.

Pour mettre en évidence au moins quelques-uns des facteurs extralinguistiques mentionnés par Angelelli (2004) nous voudrions revenir à la situation du Mexique, pays dans lequel les statuts des langues indigènes et des interprètes en milieu social sont liés à la situation politique, économique et sociale.

La politique linguistique du gouvernement mexicain a changé en fonction de sa perception de l' "intégration " de la population indigène après la Révolution de 1910. L'indigénisme, l'éducation bilingue et biculturelle et l'éducation interculturelle la plus récente résultent d'approches mises en œuvre au cours des six dernières décennies avec des résultats polémiques, que nous ne discuterons pas ici. Il faut quand même souligner qu'en dépit des politiques linguistiques et culturelles inclusives, l'espagnol reste la langue privilégiée, voire hégémonique. Elle jouit du statut qu'aucune langue indigène n'atteindra jamais, non seulement à cause de leur «faiblesse » se manifestant en chiffres et par une discrimination plus ou moins dissimulée, mais également à cause de la compétition entre différentes variantes dialectales pour la reconnaissance desquelles luttent les natifs. Ils cherchent la reconnaissance de leur variante comme la plus importante par la demande, entre autres, de formations en interprétation et médiation interculturelle. Ces formations qui engendrent des frais pour engager des enseignants et un personnel technique, sans compter la location des installations indispensables, impliquent, à leur tour, une planification économique. Finalement, la formation est aussi une question sociale, surtout au moment de définir le profil des candidat(e)s dont la connaissance avancée des langues vernaculaires, non enseignées dans la majorité des cas au niveau plus élevé que celui de l'école primaire, laisse parfois beaucoup à désirer. À tout cela, il faut ajouter encore un facteur social non négligeable, à savoir : la discrimination linguistique et raciale, illustrant l'asymétrie culturelle et sociale, toujours tangible au Mexique comme l'une des conséquences de l'époque coloniale (Kasperska, 2016b). Les rapports de force mentionnés par Foucault (1976) sont parfaitement visibles dans le contexte mexicain où les tensions interinstitutionnelles, interethniques et entre les classes sociales pèsent sur le « décor» dans lequel se joue aussi le pouvoir de l'interprète.

\footnotetext{
${ }^{5}$ " If the communication is to succeed, she must also be attentive to the social factors affecting communication, such as the social background of the interlocutors, or the constraints imposed by the setting in which the interpreted communicative event (ICE) takes place» (Angelelli, 2004, p. 7).
} 
L'exemple que nous venons de décrire met en exergue les aspects politiques, sociaux et économiques de l'interprétation en milieu social. Nous tenons à présenter maintenant la situation dans laquelle on cherche à inciter l'interprète à s'allier avec le commanditaire ou le client. Les codes de déontologie ou d'éthique ne précisent pas comment les interprètes doivent se comporter dans les situations où ils sont explicitement invités à prendre parti. Anne Martin et Isabel Abril Martí (2008) se demandent si l'interprète représente son client ou son employeur. Et nous aimerions ajouter encore un doute : envers qui faut-il finalement être loyal si le même client nous demande d'être loyaux envers une tierce personne, tout en rompant ce «contrat » au cours de l'interprétation ? Pour des raisons de confidentialité, nous ne pouvons pas dévoiler plus de détails sur le contexte en question, mais il vaut la peine d'indiquer la nature de l'interaction dans une telle situation. Cette alliance forcée, non désirée par l'interprète, exige la mise en œuvre d'une censure des répliques non adressées directement à l'autre partie, mais dont le contenu «négatif » la regarde : l'apparence physique et la modeste participation à l'entretien à caractère plutôt sociable. Le dilemme éthique consiste justement à devoir censurer le discours et à savoir comment le faire et quelles parties de l'interaction il faut retenir entre les différents participants, sans les transmettre dans l'autre langue. Nous devons admettre que nos décisions concernant l'application de la censure sont prises en fonction du dommage que les commentaires non légitimes et mal à propos peuvent causer dans la communication bien que, autant que nous sachions, aucun code d'éthique professionnelle ne désigne la censure comme un procédé de transfert. En essayant de résoudre les problèmes traductologiques, les interprètes transgressent les règles systématiquement et consciemment et cherchent à le légitimer (Wadensjö, 1995, p. 116). La censure est aussi mentionnée par Tryuk (2004) mais dans le contexte des rôles exercés par l'interprète : il devient censeur lorsqu'il décide d'éliminer les mots vulgaires prononcés par le client, c'est-à-dire, il recourt à la stratégie appelée adaptation. Dans l'exemple décrit, la seule solution possible est de s'adapter aux circonstances et d'omettre les commentaires mal à propos. Par ailleurs, il y a des codes de comportements, appelés couramment «savoir-vivre », que l'on peut mettre à profit dans les situations de communication interpersonnelle.

La censure est très proche de l'omission comme technique d'interprétation. Cecilia Wadensjö (1998, pp. 203-204) cite un exemple dans lequel l'interprète avoue avoir omis un énoncé prononcé par un réfugié arménien dans un camp au Danemark, pour lui épargner une situation embarrassante devant une fonctionnaire de l'État. Il s'agissait d'un propos sur l'introduction du christianisme en Arménie en 301 av. J.-C. En omettant cet énoncé l'interprète a pris en considération deux facteurs : le statut de l'homme en tant que père de famille et membre de la communauté catholique arménienne, d'une part, et les attentes de la fonctionnaire, de l'autre. Wadensjö (1998) souligne que les présuppositions de l'interprète à propos de la réaction d'une des parties peuvent être incompatibles avec la réaction réelle, comme 
cela s'est avéré dans l'exemple cité, après la vérification des objectifs des questions posées par la fonctionnaire. Ce que Wadensjö suggère c'est une réflexion sur le «cadre de références » $(1998$, p. 205) qui n'est pas forcément le même pour l'institution et le client. En fait, il y a trois cadres de références parce que celui de l'interprète engagé doit être, lui aussi, pris en considération.

Des situations semblables à celle analysée par Wadensjö sont assez fréquentes dans notre pratique d'interprète. Par exemple, pendant un évènement culturel, un diplomate a avoué avoir visité la plus importante université de la région, en confondant son nom avec une autre université polonaise. Sachant qu'en réalité il avait rendu visite à l'université de la ville où l'on se trouvait, nous avons corrigé son nom pour ne pas ridiculiser la partie française et épargner la confusion parmi les personnes présentes à l'évènement ainsi que pour maintenir la logique du discours. Dans un autre contexte, nous avons changé le nom propre de « Nowy Meksyk » (un état des États-Unis de l'Amérique du Nord), utilisé par un haut fonctionnaire polonais devant la délégation mexicaine, par celui de «Nuevo León» (un état du Mexique) d'où ladite délégation venait. À part le fait qu'il s'agisse d'un lapsus linguistique, la confusion entre un état mexicain et un état américain aurait pu signifier une mésestime du pays invité. Dans les deux cas, les changements ont été dictés par le «facteur local» de coordination de l'entretien (Wadensjö, 1998, p. 203), pour éviter des malentendus et des réactions indésirables.

Le dernier cas que nous avons l'intention de présenter, est le caractère métalinguistique de la communication et le langage corporel qui l'accompagnent. Ce sont des comportements qui sont à la fois interpersonnels, sociaux et linguistiques. En interprétation communautaire, ils se manifestent très souvent lorsque les participants à l'interaction ne sont pas familiers avec les principes et les règles de l'interprétation. Au lieu de s'adresser directement au client ou au récepteur, selon le cas, ils s'adressent à l'interprète. Dans notre pratique professionnelle, nous avons eu affaire à de multiples exemples de commentaires métalinguistiques de la part des émetteurs, à savoir : "et maintenant c'est à vous d'interpréter », « expliquez-lui que... » ou « vous n'êtes pas obligée d'interpréter tous les détails », pour ne citer que quelques exemples parmi les plus fréquents. Ces formules métalinguistiques sont caractéristiques de l'interprétation en milieu social parce que l'interprète participant à l'interaction est tout simplement visible. Pour cette raison, très souvent, les intervenants non seulement lui parlent, mais se tournent aussi vers lui et le regardent, ou encore font des gestes et des grimaces dirigés vers lui au lieu de traiter le récepteur comme le « vrai » partenaire : l'interlocuteur dans la communication. Tryuk (2004) considère ces énoncés comme une preuve de l'inclusion de l'interprète parmi les interlocuteurs. Lorsqu'on lui « propose des services qui dépassent sa fonction primaire de rendre fidèlement le message entendu » (Tryuk, 2004, p. 28), par exemple en lui demandant de faire un résumé du message entendu, c'est déjà une ingérence dans la prestation de service d'interprétation. En conclusion, ces comportements verbaux et 
non verbaux, tout à fait naturels et spontanés chez les participants « non professionnels » à l'interaction, mettent en évidence, en premier lieu, l'inclusion de l'interprète dans l'échange, faite « au détriment » du récepteur qui peut se sentir exclus. En second lieu, certaines formules métalinguistiques réduisent le pouvoir de l'interprète en lui imposant, par exemple, des techniques de transfert.

Selon les résultats de la recherche réalisée par Martin et Abril Martí (2008) auprès d'interprètes communautaires en Espagne, ceux-ci se sont avérés comme intervenant librement, adaptant les énoncés, ajoutant des explications sur les aspects culturels et proportionnant les informations relatives au fonctionnement des services publiques. Mais, ce que les chercheuses ont également observé, c'est que les interprètes essaient de ne pas omettre ou résumer l'information. Normalement, ils interviennent en fonction des besoins du client qui ne parle pas espagnol parce que c'est à lui qu'ils s'identifient. La recherche menée en Espagne a dévoilé aussi que c'est sur l'intuition que le travail des interprètes est basé parce que ceux-ci manquent de formation et sont très souvent autodidactes. Ils se servent de critères et de leur expérience personnels, bref, de leur intuition professionnelle (Martin \& Abril Martí, 2008). Invités à énumérer trois traits caractéristiques de l'interprétation de qualité, ils ont indiqué les suivants: la patience, la connaissance des langues de travail, l'empathie, l'amabilité, la propension à aider, la connaissance des cultures en question, le savoir écouter, la capacité de communiquer et de s'exprimer clairement, la responsabilité, la connaissance du contexte de travail, la concision et l'impartialité. Pour les traits caractéristiques inclus dans les codes de déontologie Martin et Abril Martí ont employé les italiques que nous reproduisons ici. Dans ce portrait des interprètes en Espagne, on peut observer que, même en refusant d'omettre ou de résumer l'information, c'est-à-dire de recourir à des techniques d'interprétation légitimes, ils s'engagent dans l'interaction en s'identifiant aux usagers étrangers des services sociaux en Espagne. Et c'est en cela que leur pouvoir se manifeste : le choix de servir le client, le plus souvent vulnérable face aux institutions de l'État.

\section{CONCLUSION}

Les situations que nous avons présentées ne sont qu'une sélection d'exemples qui nous ont servi à illustrer les aspects les plus difficiles du travail d'interprète en milieu social. À notre avis, la difficulté majeure consiste à mesurer le pouvoir là où les enjeux de la communication dépassent le simple cadre de l'échange interlinguistique et interculturel.

L'analyse des situations choisies a démontré que le pouvoir de l'interprète est dynamique, en changeant d'une interaction à l'autre. Ce pouvoir sur le déroulement de la communication peut être réel lorsque la censure ou l'engagement personnel sont impliqués, que leur influence soit positive ou négative. Le pouvoir de l'inter- 
prète (interprète-employé de l'institution) est manifestement diminué parce que son double rôle fait que le pouvoir se dilue en «loyautés». À vrai dire, il est difficile d'imaginer une situation où le pouvoir de l'interprète soit complètement éliminé, si on le considère comme un participant réel à la communication, censé être une personne responsable et respectant le secret professionnel. C'est à ce niveau déjà que la neutralité de l'interprète se termine.

Comme il est impossible de prévoir le déroulement de toutes les situations communicationnelles et les réactions des intervenants en interprétation communautaire (Tryuk, 2004, 2006), les rapports de force et les limites du pouvoir de l'interprète ne sont pas définis (Springer, 2010) dans les codes de déontologie. En tant qu'enseignante d'interprétation et co-auteure d'un programme de formation des médiateurs, traducteurs et interprètes en milieu social mis en œuvre en 2014 à l'Université Interculturelle de l'État de Veracruz incluant l'éthique et la responsabilité professionnelle (Kasperska, 2016b) comme matières obligatoires, nous avouons que l'aspect éthique dans la préparation professionnelle à l'université, aussi bien dans le cas des interprètes que des traducteurs, est généralement négligé. Katarzyna Kruk-Junger (2013) observe que la formation professionnelle ne prépare pas les interprètes communautaires à exercer la profession, bien au contraire : elle risque de s'avérer inutile, voire obstructive pour la communication dans les situations réelles d'interprétation en milieu social. Nous pensons qu'il est indispensable de sensibiliser les étudiants et les adeptes de l'interprétation en leur montrant les enjeux éthiques qui se manifestent inévitablement dès la première tâche.

Pareillement à Kruk-Junger (2013), Moira Inghilleri (2005) met en relief le fait que, dans la pratique professionnelle, les interprètes en services sociaux se retrouvent dans des situations «contradictoires» par rapport à la formation reçue et les normes qu'ils ont appris à observer. Parmi les conflits entre la disposition de l'interprète et celle des autres participants à l'entrevue (demandeurs d'asile et fonctionnaires de l'État) elle énumère les différentes attentes face au type et à la mesure des interventions de l'interprète, qui peuvent concerner la culture et la langue du demandeur d'asile, les normes du processus de la demande d'asile et la diversité des codes des usages acquis par l'interprète au cours de la formation ou au sein des agences de traductions.

En s'appuyant sur la formation ou l'expérience acquises, les interprètes font des choix stratégiques qui reflètent leurs attitudes face au code déontologique, c'est-à-dire, ils le suivent au pied de la lettre ou s'en distancient en rejetant les exigences qu'ils trouvent «non pertinentes ou irréalisables» (Inghilleri, 2005, p. 7). L'engagement personnel qu'ils s'imposent par eux-mêmes à leurs interlocuteurs, négocié avec eux ou provoqué par eux, peut bouleverser les relations de pouvoir préétablies. On souligne très souvent qu'en services sociaux, le déroulement de 
l'échange est imprévisible. Aussi les relations de force entre les participants s'éclaircissent-elles au cours de la rencontre. Nous considérons qu'en raison de ces deux caractéristiques, le degré de pouvoir de l'interprète doit se négocier également pendant la réalisation de la tâche et pas seulement avant. En ce qui concerne la transgression des limites déontologiques qui lui sont imposées en théorie, elle devrait être re(vue) dans des contextes concrets, c'est-à-dire, dans le cadre de références de tous les participants du trialogue. 


\section{RÉFÉRENCES BIBLIOGRAPHIQUES}

Angelelli, C.V. (2003). «The Interpersonal Role of the Interpreter in Cross-Cultural Communication: A Survey of Conference, Court and Medical Interpreters in the US, Canada and Mexico ». In L. Brunette, G.L. Bastin, I. Hemlin \& H. Clarke (réd.), Interpreters in the Community: Selected Papers from the Third International Conference on Interpreting in Legal, Health and Social Service Settings, Montréal, Québec, Canada 22-26 May 2001 (pp. 15-26). Amsterdam/Philadelphia : John Benjamins Publishing Company. DOI : 10.1075/btl.46.06ang.

Angelelli, C.V. (2004). Revisiting the Interpreter's Role. A study of conference, court, and medical interpreters in Canada, Mexico, and the United States. Amsterdam/Philadelphia : John Benjamins Publishing Company.

Antonini, R. (2011). « Natural translator and interpreter ». In Y. Gambier \& L. van Doorslaer (réd.), Handbook of translation. Vol. 2 (pp. 102-104). Amsterdam/Philadelphia : John Benjamins Publishing Company.

Barsky, R.F. (2005). «Activist Translation in an Era of Fictional Law1 ». TTR : traduction, terminologie, rédaction, 18 (2), 17-48. DOI : 10.7202/015745ar.

Buzelin, H. (2011). « Agents of translation ». In Y. Gambier \& L. van Doorslaer (réd.), Handbook of translation. Vol. 2 (pp. 6-12). Amsterdam/Philadelphia : John Benjamins Publishing Company. DOI : 10.1075/hts.2.age1.

Centro Prodh. (2018). "Caso de Doña Jacinta Francisco Marcial ». Accessible sur le site http://www.centroprodh.org.mx/index.php?option=com_content\&view=category\&id=237\&la yout=blog\&Itemid=196\&lang=es. Dernière consultation le 13 mai 2018.

Foucault, M. (1976). Histoire de la sexualité. Vol. 1 : La volonté de savoir. Paris : Gallimard.

Harris, B. (1976). « The Importance of Natural Translation ». Working Papers in Bilingualism, 12, 96-114.

INEGI (2015). « Lengua indígena ». Accessible sur le site http://www.inegi.org.mx/. Dernière consultation le 13 mai 2018.

Inghilleri, M. (2005). « Mediating Zones of Uncertainty ». The Translator, vol. 11, no. 1, 1-17.

Kasperska, I. (2015). «Badania przekładoznawcze i kształcenie tłumaczy w Meksyku ». Rocznik Przekładoznawczy. Studia nad teoria, praktyka i dydaktyka przektadu, 10, 271-291. DOI : 10.12775/RP.2015.017.

Kasperska, I. (2016a). « Myślenie pograniczne albo tłumaczenie w kontekście hybrydowym ». In K. Kodeniec \& J. Nowacka (réd.), Komunikacja międzykulturowa w świetle wspótczesnej translatologii. Vol. V : Język przektadu i komunikacji międzykulturowej (pp. 63-74). Olsztyn : Katedra Filologii Angielskiej UWM w Olsztynie.

Kasperska, I. (2016b). « Kształcenie tłumaczy języków narodowych w Meksyku w kontekście asymetrii językowo-kulturowej ». In B. Walkiewicz, A. Fimiak-Chwiłkowska \& J. Woroch (réd.), Norma a uzus I. Z zagadnień przekładu specjalistycznego (pp. 155-165). Poznań : Wydawnictwo Naukowe UAM.

Kleinert, C.V. (2016). Formación e iniciación profesional de intérpretes de lenguas nacionales mexicanas para la justicia : el caso de Puebla. Dissertation doctorale non publiée, défendue en juin 2016 à l'Université de Veracruz, Xalapa, Mexique.

Klimkiewicz, A. (2005). «L'interprétation communautaire : un modèle de communication trialogique ». TTR : traduction, terminologie, rédaction, 18 (2), 209-224. DOI : 10.7202/015771ar.

Kruk-Junger, K. (2013). Przekład ustny środowiskowy. Teoria, normy, praktyka. Bielsko-Biała : Akademia Techniczno-Humanistyczna w Bielsku-Białej.

Määttä, S.K. (2017). «English as a Lingua Franca in telephone interpreting: representations and linguistic justice ». The Interpreters' Newsletter, 22, 39-56. DOI : 10.13137/2421-714X/20737. 
Martin, A. \& Abril Martí, I. (2008). "Community interpreter self-perception. A Spanish case study ». In A. Martin \& C. Valero-Garcés (réd.), Crossing Borders in Community Interpreting. Definitions and Dilemmas (pp. 203-230). Amsterdam/Philadelphia : John Benjamins Publishing Company.

Pöchhacker, F. (2004). Introducing Interpreting Studies. London/New York : Routledge.

Sadan, E. (1997). Empowerment and Community Planning. Trad. de l'hébreu par R. Flantz. Accessible sur le site http://www.mpow.org. Dernière consultation le 9 septembre 2018.

Springer, R. (2010). « Rola(e) tłumacza środowiskowego w kontekście azylanckim i uchodźczym a etyka, standardy zawodowe i oczekiwania pracowników polskiego urzędu imigracyjnego ». In M. Tryuk (réd.), O tlumaczach, prawnikach, lekarzach i urzędnikach. Teoria i praktyka thumaczenia środowiskowego w Polsce (pp. 157-215). Warszawa : BEL Studio Sp. z o.o.

Tryuk, M. (2004). L'Interprétation communautaire. Des normes et des rôles dans l'interprétation. Warszawa : Wydawnictwo TEPIS.

Tryuk, M. (2006). Przekład ustny środowiskowy. Warszawa : Wydawnictwo Naukowe PWN.

Tymoczko, M. (2007). Enlarging Translation, Empowering Translators. Manchester/Kinderhook (NY) : St. Jerome Publishing.

Valero-Garcés, C. \& Martin, A. (2008). «Introduction ». In C. Valero-Garcés \& A. Martin (réd.), Crossing Borders in Community Interpreting. Definitions and Dilemmas (pp. 1-7). Amsterdam/Philadelphia : John Benjamins Publishing Company.

Wadensjö, C. (1995). « Dialogue Interpreting and the Distribution of Responsibility ». Hermes, Journal of Linguistics, 14, 111-129.

Wadensjö, C. (1998). Interpreting as Interaction. London/New York : Longman. 